OPEN ACCESS

Edited by:

Zhi-Ping Liu,

Shandong University, China

Reviewed by:

Milind B. Ratnaparkhe,

ICAR Indian Institute of Soybean

Research, India

Renxiang Yan,

Fuzhou University, China

${ }^{*}$ Correspondence:

Changfei Guan

guanchangfei@nwafu.edu.cn

Shuyuan Liu

shuyuan_liu@nwafu.edu.cn

tThese authors have contributed equally to this work

Specialty section

This article was submitted to

Computational Genomics,

a section of the journal

Frontiers in Genetics

Received: 18 February 2021

Accepted: 06 April 2021

Published: 29 April 2021

Citation:

Chachar S, Liu J, Zhang P, Riaz A,

Guan C and Liu S (2021) Harnessing

Current Knowledge of DNA

N6-Methyladenosine From Model

Plants for Non-model Crops.

Front. Genet. 12:668317.

doi: 10.3389/fgene.2021.668317

\section{Harnessing Current Knowledge of DNA N6-Methyladenosine From Model Plants for Non-model Crops}

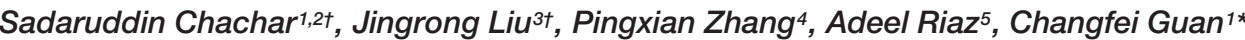 \\ and Shuyuan Liü*
}

1 State Key Laboratory of Crop Stress Biology for Arid Areas, College of Horticulture, Northwest A\&F University, Yangling, China, ${ }^{2}$ Department of Biotechnology, Faculty of Crop Production, Sindh Agriculture University, Tandojam, Pakistan, ${ }^{3}$ College of Mathematics and Statistics, Northwest Normal University, Lanzhou, China, ${ }^{4}$ Biotechnology Research Institute, Chinese Academy of Agricultural Sciences, Beijing, China, ${ }^{5}$ Deaprtment of Biochemistry, Faculty of Life Sciences, University of Okara, Okara, Pakistan

Epigenetic modifications alter the gene activity and function by causing change in the chromosomal architecture through DNA methylation/demethylation, or histone modifications without causing any change in DNA sequence. In plants, DNA cytosine methylation $(5 \mathrm{mC})$ is vital for various pathways such as, gene regulation, transposon suppression, DNA repair, replication, transcription, and recombination. Thanks to recent advances in high throughput sequencing (HTS) technologies for epigenomic "Big Data" generation, accumulated studies have revealed the occurrence of another novel DNA methylation mark, N6-methyladenosine $(6 \mathrm{~mA})$, which is highly present on gene bodies mainly activates gene expression in model plants such as eudicot Arabidopsis (Arabidopsis thaliana) and monocot rice (Oryza sativa). However, in non-model crops, the occurrence and importance of $6 \mathrm{~mA}$ remains largely less known, with only limited reports in few species, such as Rosaceae (wild strawberry), and soybean (Glycine max). Given the aforementioned vital roles of $6 \mathrm{~mA}$ in plants, hereinafter, we summarize the latest advances of DNA 6mA modification, and investigate the historical, known and vital functions of $6 \mathrm{~mA}$ in plants. We also consider advanced artificial-intelligence biotechnologies that improve extraction and prediction of $6 \mathrm{~mA}$ concepts. In this Review, we discuss the potential challenges that may hinder exploitation of $6 \mathrm{~mA}$, and give future goals of $6 \mathrm{~mA}$ from model plants to non-model crops.

Keywords: epigenetic modification, DNA methylation, N6-methyladenosine, gene expression, artificial intelligence, deep learning

\section{INTRODUCTION}

Hereditary changes in activity and function of a gene caused by the direct alteration of DNA sequence like deletions, insertions, point mutations and translocations is known as Genetics. While epigenetics is the phenomenon of heritable changes in the activity or function of a gene without causing any change in DNA sequence itself (Heard and Martienssen, 2014; Allis and Jenuwein, 2016). The epigenetic mechanism mainly including histone posttranscriptional modifications (PTMs) and DNA methylation plays a vital role in gene expression and various biological functions (Heard and Martienssen, 2014; Allis and Jenuwein, 2016). In consideration of epigenetic enzymatic 
systems, for dynamically installing, removing, and epigenetic modifications; epigenetic regulation is installed by "writers," removed by "erasers", and read by "readers" with a battery of proteins to facilitate biochemical modifications on DNA and histone proteins (Mozgova and Hennig, 2015; Allis and Jenuwein, 2016). Recently, it has enabled the advancement in breeding approaches providing new source of variability originating from epi-alleles (Gallusci et al., 2017; Springer and Schmitz, 2017).

DNA methylation is another most commonly studied epigenetic mark that is responsible for regulating numerous cellular pathways along with gene expression (Feng et al., 2010; Smith and Meissner, 2013). From the genomic DNA of diverse species, numerous methylated bases have been reported like 5-methylcytocine $(5 \mathrm{mC})$, N4-methylcytocine $(4 \mathrm{mC})$, and N6methyladenine $(6 \mathrm{~mA})$. As a well-studied repressive mark, $5 \mathrm{mC}$ has shown dynamic regulation of establishing, maintaining, and active removal of their activities, and also involved in gene silencing, transposon insertions, deletion, genome stability and gene expression regulation during all progressive stages in plants (Niederhuth and Schmitz, 2017; Gallego-Bartolomé et al., 2018). Recent reports have revealed that DNA methylation performs a significant role in controlling the growth, development and ripening in horticultural plants; During plant growth, $5 \mathrm{mC}$ levels show tissue specific features while during development the methylation levels exhibits variations (Lang et al., 2017; Cheng et al., 2018; Huang et al., 2019; Sabbione et al., 2019; Liang et al., 2020; Tang et al., 2020).

As DNA 5mC and other histone modifications have previously been discussed in other reviews in non-model crops (Duan et al., 2018; Tang et al., 2020), now we merely focus on the comparatively uncharacterized DNA $6 \mathrm{~mA}$ methylation in plants. In this Review, we introduce the dynamic distribution pattern and motifs of $6 \mathrm{~mA}$ in plants. Recent advances in high-throughput sequencing (HTS) technologies for detecting $6 \mathrm{~mA}$, we have also summarized the $6 \mathrm{~mA}$ methyltransferases (writers) and demethylases (erasers), the molecular and biological functions of $6 \mathrm{~mA}$ in plants. We also consider advanced artificial-intelligence biotechnologies that improve extraction and prediction of $6 \mathrm{~mA}$ concepts. Finally, we discuss the potential challenges that may hinder exploitation of $6 \mathrm{~mA}$, and in the end we have given future perspectives of $6 \mathrm{~mA}$ from model plants to non-model crops.

\section{6mA DETECTION METHODS IN PLANTS}

Detection of DNA methylation has evolved over the years to become progressively accurate and sensitive. In plants, various methods have been developed for $6 \mathrm{~mA}$ detection, such as Dot blot, high-performance liquid chromatography combined with mass spectrometry (HPLC-MS/MS), methylated DNA immunoprecipitation sequencing (MeDIP-seq) for $6 \mathrm{~mA}$ (6mA-IP-seq), 6mA-IP-seq combining photo-crosslinking with exonuclease digestion (6mA-CLIP-Exo-seq), and methylated DNA with restriction enzyme digestion followed by sequencing (6mA-RE-seq), and third-generation high-throughput sequencing (HTS) technologies including single-molecule real time sequencing (SMRT-seq) and Nanopore sequencing (Nanopore-seq) (Figure 1A; Liang et al., 2020).

HPLC-MS/MS has been used for detecting the lowest levels of up to $0.00001 \% 6 \mathrm{~mA}$ in the genome of different organisms (Huang et al., 2015). An alternative approach, dot blot assay with specific $6 \mathrm{~mA}$ antibodies was applied, but with limitation of detection resolution (Liang et al., 2020). These two detection methods have been successfully used for the presence of $6 \mathrm{~mA}$ in certain organisms, but they are unable to provide genomic location of $6 \mathrm{~mA}$.

For determining the genome wide $6 \mathrm{~mA}$ sites, numerous methylation sensitive sequencing approaches have been established. Firstly, 6mA-IP-seq and its related 6mA-CLIP-Exoseq can achieve near single-nucleotide resolution of $6 \mathrm{~mA}$ (Chen et al., 2015). However, 6mA-IP-seq has also limitations; it is antibody dependent precisely recognizing $6 \mathrm{~mA}$. For example, the antibody used to detect $6 \mathrm{~mA}$ can also detect $\mathrm{m}^{6} \mathrm{~A}$ in eukaryotic mRNAs (Luo et al., 2014); thus these antibody approaches are not quantitative and maybe confounded by recognition of other adenine modifications (such as $\mathrm{m}^{6} \mathrm{~A}$ and $\mathrm{m}^{1} \mathrm{~A}$ in RNA), and require pure DNA with RNase enzyme digestion.

Another method for detecting 6mA-RE-seq involves radioactive methylation of DNA coupled with restriction digestion, electrophoresis and sequencing ( $\mathrm{Fu}$ et al., 2015; Liang et al., 2020). Digestion of methylated DNA with the restriction enzymes such as $D p n \mathrm{I}$ (recognize $5^{\prime}$-G6mATC-3'), DpnII (recognize unmethylated $5^{\prime}$-GATC- $3^{\prime}$ ), and CviAII (recognize unmethylated $5^{\prime}$-CATG-3') can accurately detect and identify $6 \mathrm{~mA}$ sites at single-base resolution (Liang et al., 2020). But 6mA-RE-seq is limited in detection of specific sequence motifs with relative low proportions of $6 \mathrm{~mA}$ in the whole genome.

Single-molecule real time sequencing (SMRT-seq) is a third generation sequencing technique, it offers accurate sequence reads and measures the kinetic rate of nucleotide incorporation during sequencing (Flusberg et al., 2010). SMRT sequencing has been used to map $6 \mathrm{~mA}$ and $5 \mathrm{mC}$ simultaneously in Escherichia coli (Fang et al., 2012) 6mA in Caenorhabditis elegans (Greer et al., 2015) and 6mA in green algae and human lymphoblastoid cells (hLCLs) (Zhu et al., 2018). SMRT can detect every DNA modification at single base resolution (Zhu et al., 2018). Recently, $6 \mathrm{~mA}$ has been detected on genome-wide level in eudicot model Arabidopsis thaliana (Liang et al., 2018b) and monocot model rice (Oryza sativa) (Zhang et al., 2018; Zhou et al., 2018), as well as in non-model plants Casuarina equisetifolia (Ye et al., 2019), woodland strawberry (Fragaria vesca) (Xie et al., 2020), and soyabeans (Glycine max) (Yuan et al., 2020), and fig (Ficus carica) (Usai et al., 2021). While combined SMRT sequencing and $6 \mathrm{~mA}$-IP-Seq have been used to detect genome wide $6 \mathrm{~mA}$ levels in Arabidopsis and rice (Liang et al., 2018b; Zhang et al., 2018; Zhou et al., 2018), and they reveals more efficient information for dynamic distribution and pattern of $6 \mathrm{~mA}$ compared to these $6 \mathrm{~mA}$ studies in non-model plants. In addition, in silico database for storing genome-wide $6 \mathrm{~mA}$ information have also been developed for integrative $6 \mathrm{~mA}$ and other DNA modifications in both rice and Rosaceae from SMRT sequencing datasets (Liu Z. Y. et al., 2019; Zhang et al., 2020). 

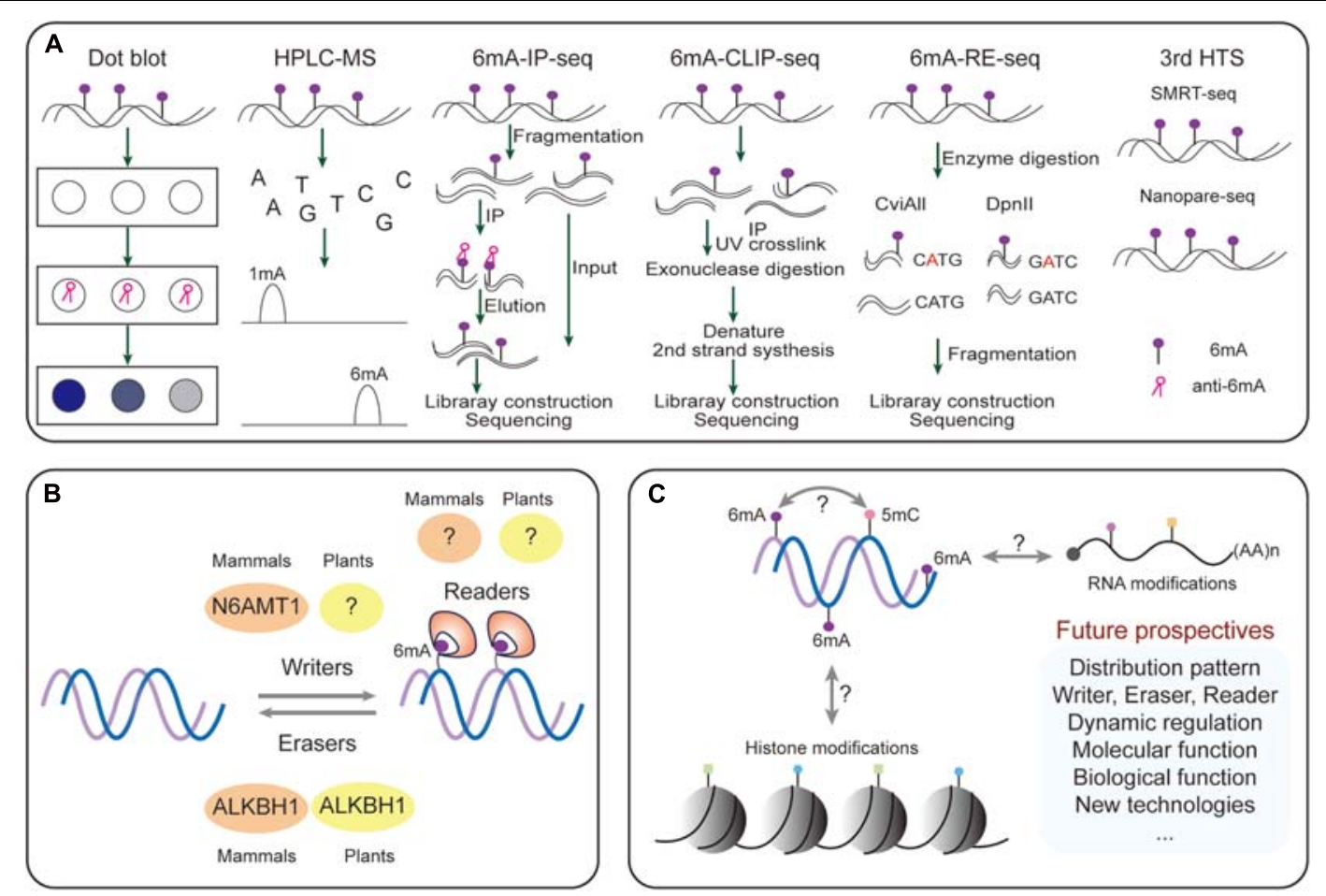

FIGURE 1 | The dynamic DNA 6mA modification in plants. (A) Methods for detecting 6mA. (B) The enzymatic systems of $6 \mathrm{~mA}$. (C) Potential association with other epigenetic modifications and future perspective of $6 \mathrm{~mA}$. In plants, $6 \mathrm{~mA}$ can be detected by various methods, such as Dot blot, HPLC-MS, 6mA-IP-seq, 6mA-CLIP-Exo-seq, 6mA-RE-seq, SMRT-seq, and Nanopore-seq. The first two can only detect global 6mA with both qualitative and quantitative analyses but not detect specific 6mA sites; 6mA-IP-seq, 6mA-CLIP-Exo-seq, and 6mA-RE-seq can decipher genome-wide 6mA sites or peaks at a large scale. While, SMRT-seq and Nanopore-seq can identify single-base resolution $6 \mathrm{~mA}$ and illustrate computational motifs and distribution patterns for the accuracy and robustness of $6 \mathrm{~mA}$ detection. Currently, we are only known that N6AMT1 is a 6mA writer in mammals, ALKBH1 acts as both erasers in mammals and plants; it remains unknown the readers for recognizing $6 \mathrm{~mA}$ site on the genome.

For the genomes having lower DNA abundance modifications, the sensitivity and specificity of detection methods need to be balanced. High sensitivity but low specificity may yield false positive results, while weak signals may be missed with high specificity but low sensitivity. To achieve relatively high sensitivity and high specificity, multi-strategy cross-validation is essential in order to detect a rare DNA modification. A combination of complimentary techniques is ideal for convincingly detecting rare modifications such as $6 \mathrm{~mA}$, as each technique has its own limits.

\section{DNA 6mA METHYLTRANSFERASES AND DEMETHYLASES IN PLANTS}

In DNA the formation of $6 \mathrm{~mA}$ takes place by recognition of respective adenine by specific enzymatic systems, including methyltransferases (writers), demethylases (erasers) and DNA $6 \mathrm{~mA}$ methylation binding proteins (readers). In mammals the AlkB family proteins have also been found to act as $6 \mathrm{~mA}$ demethylase (Figure 1B; Liang et al., 2020). Recently the rice homologous gene OsALKBH1 has been identified (Zhou et al., 2018). For further confirming the function of OsALKBH1 in demethylation two mutant lines were obtained by Clustered
Regularly Interspaced Short Palindromic Repeats (CRISPR)associated protein 9 (Cas9). No visible variation was observed in phenotype of these lines except earlier heading displayed two to threefold increase in $6 \mathrm{~mA}$ levels. While, knockout of OsALKBH1 did not display any effect on total $5 \mathrm{mC}$ levels, suggesting that overall $5 \mathrm{mC}$ levels remained unaffected with the increase in $6 \mathrm{~mA}$ levels in rice genome (Zhou et al., 2018). The OsALKBH1 protein has been shown to be localized in the cytoplasm and nucleus (Zhou et al., 2018), and the putative orthologs of ALKBH1 proteins may be well conserved across plant kingdoms (Figures 2A,B). Remarkably, protein structure modeling shows that ALKBH1 putative orthologs are well conserved in rice, Arabidopsis, soybean and strawberry (Figure 2C). Further observation along with its demethylase activity on ssDNA indicates that OsALKBH1 may carryout $6 \mathrm{~mA}$ demethylation from both RNA and DNA (Zhou et al., 2018), much like the condition in which the similar enzymes are involved methylating both $6 \mathrm{~mA}$ in DNA and $\mathrm{m}^{6} \mathrm{~A}$ in RNA (O’Brown and Greer, 2016).

Recently, N6AMT1 representing a distinct MT-A70 methyltransferase was characterized in mammals (Xiao et al., 2018). However, although it has had orthologs of MT-A70 family proteins in plant kingdoms, it is unknown whether these proteins can act as 6mA methyltransferases in plants (Liang et al., 2020). 

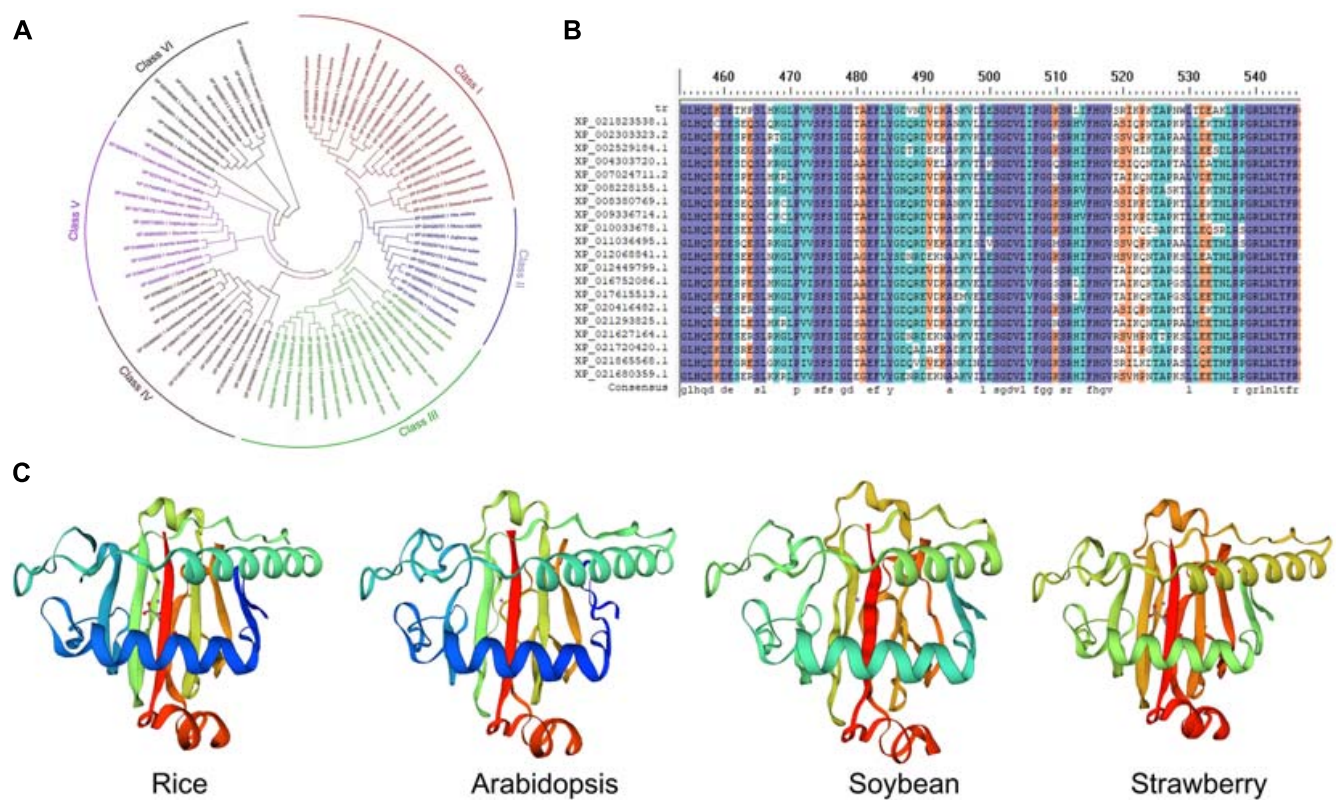

FIGURE 2 | The ALKBH1 orthologs in plants. (A) NJ tree and (B) sequence alignment of ALKBH1 orthologs in plants. (C) Protein structure of ALKBH1 orthologs in rice, Arabidopsis, soybean, and strawberry. Sequences of selected ALKBH1 putative orthologs were downloaded from the NCBI database, and then aligned. Phylogenetic tree was established by MEGA7 using neighbor-joining (NJ) tree with 1,000 replicate bootstrap support. Homology-modeling-based structural prediction of the ALKBH1 orthologs followed by the rice ALKBH1 structure (5XEG; Zhou et al., 2018).

In addition, we are also still unknown about $6 \mathrm{~mA}$ readers in both mammals and plants. So far only OsALKBH1 in rice has been revealed as a potential $6 \mathrm{~mA}$ eraser, it would be interesting to identify and characterize other $6 \mathrm{~mA}$ writers, erasers and even readers in both model plants and non-model crops that are responsible for altering the genomic DNA.

\section{MOLECULAR FUNCTION OF $6 \mathrm{~mA}$ IN PLANTS}

Recently several studies have confirmed the genome-wide distribution of $6 \mathrm{~mA}$ in plants. Thanks to advance in $6 \mathrm{~mA}$ HTS methods as mentioned above, it allows to exam whether $6 \mathrm{~mA}$ could influence transcription by altering transcription factor binding or modifying chromatin structure or not. For example, Liang et al. measured the genome-wide distribution and levels of $6 \mathrm{~mA}$ in gDNA of Arabidopsis, applying different approaches, including DNA dot blot, using a specific $6 \mathrm{~mA}$ antibody, SMRT sequencing and LC-MS/MS, which could detect the strand specific $6 \mathrm{~mA}$ sites at single nucleotide resolution (Liang et al., 2018b). In Arabidopsis genome, $6 \mathrm{~mA}$ is present in significant levels and broadly distributed with dynamic variation in its levels at different developmental stages in different tissues, which around $30 \%$ of $6 \mathrm{~mA}$ exist on gene bodies (Liang et al., 2018b). Detected by SMRT-seq, a relatively abundant $6 \mathrm{~mA}$ sites were located on gene bodies in other plants (Zhang et al., 2018; Xie et al., 2020; Yuan et al., 2020). Within the gene bodies, about half of $6 \mathrm{~mA}$ sites were located in exons (more than $80 \%$ in exons for Arabidopsis)
(Figure 3A). Of important note, plants may share consensus sequence elements (motifs), when compared in Arabidopsis, rice, soybean and strawberry by a search for $6 \mathrm{~mA}$ consensus motifs (Figure 3B). These results may indicate conserved and essential roles of $6 \mathrm{~mA}$ in regulation of gene expression across plant species.

Remarkably, in plants and mammals, $6 \mathrm{~mA}$ has been shown to have opposite effects on the transcription factor binding. The distribution and function of $6 \mathrm{~mA}$ is found to be conserved in most of the mammalian species, $6 \mathrm{~mA}$ is found to be enriched around TSS and then actively transcribed in gene expression (Liang et al., 2020). Similar kind of phenomenon was also observed in Arabidopsis (Liang et al., 2018b) and later in rice (Zhang et al., 2018). Comparison $6 \mathrm{~mA}$ methylome and RNA sequencing data in plants showed that $6 \mathrm{~mA}$ is positively correlated with actively transcribed genes (Liang et al., 2018a,b). Thus, it seems that $6 \mathrm{~mA}$ is a general mark of active genes, though it is presently not clear whether it plays any role in the dynamic gene expression regulation. While Zhou et al. (2018) reported the $6 \mathrm{~mA}$ methylome in rice by performing $6 \mathrm{~mA}$ immunoprecipitation followed by sequencing, which revealed that $6 \mathrm{~mA}$ peaks are evenly distributed in the chromosomes and correlate with $5 \mathrm{mC}$ distribution. Contrastingly, the well-known $5 \mathrm{mC}$ is correlated with transcription repression. In addition, $6 \mathrm{~mA}$ has been shown to associate with accessible chromatin regions, $3 \mathrm{D}$ chromatin structures and other types of epigenetic modifications (e.g., histone modifications, non-coding RNAs) to regulate gene transcription in plants (Liang et al., 2021; Zhou et al., 2021). Thus, it needs more investigation on what molecular role of $6 \mathrm{~mA}$ has during transcription in plants. 


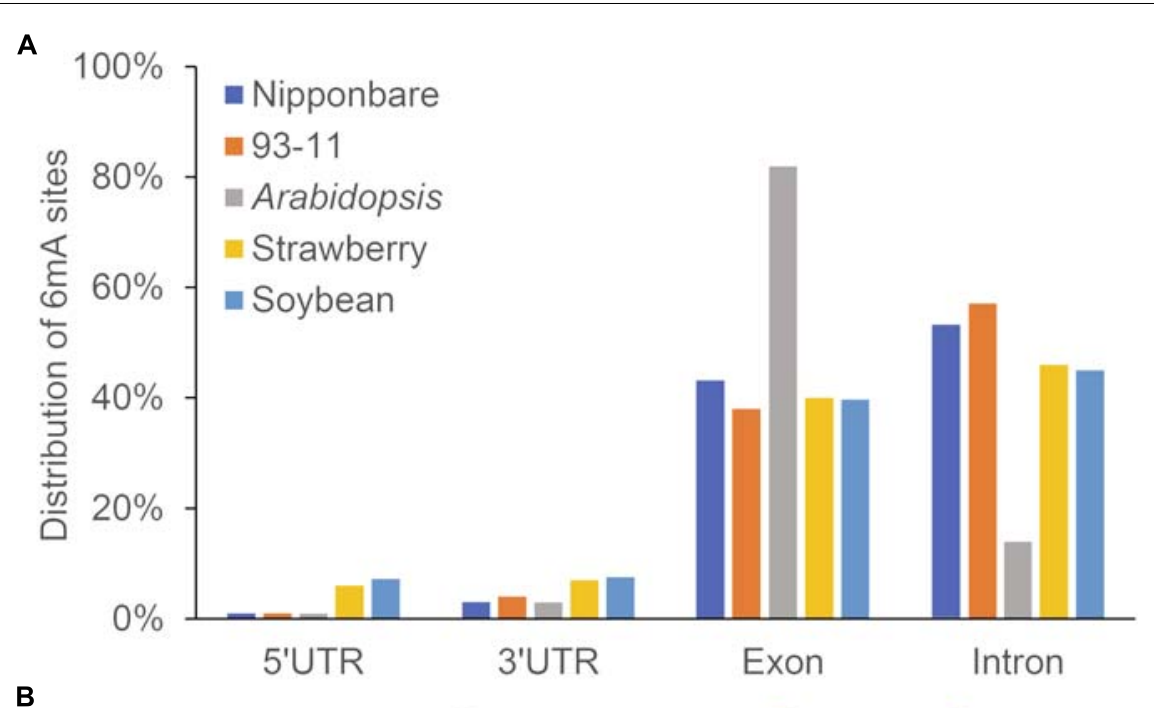

B

Rice(Nipponbare)
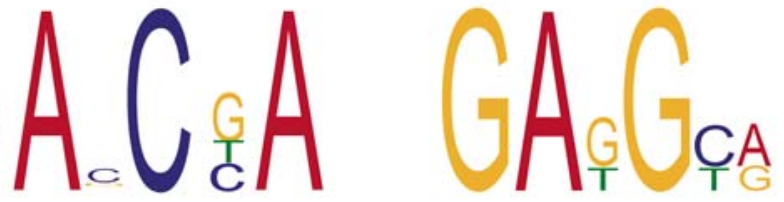

Rice (93-11)
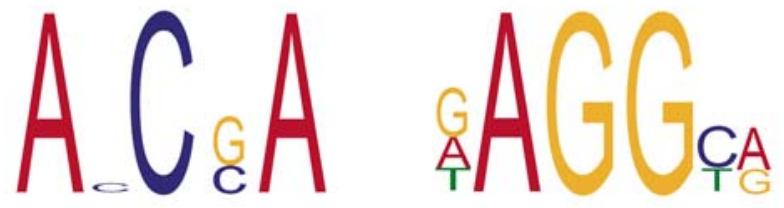

Arabidopsis
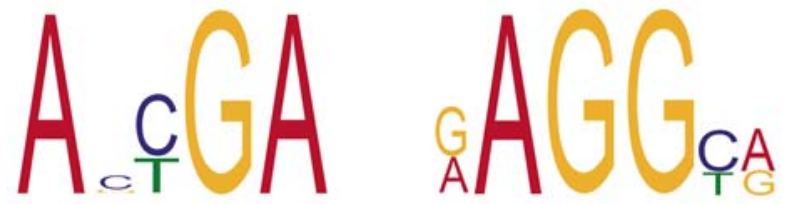

Strawberry
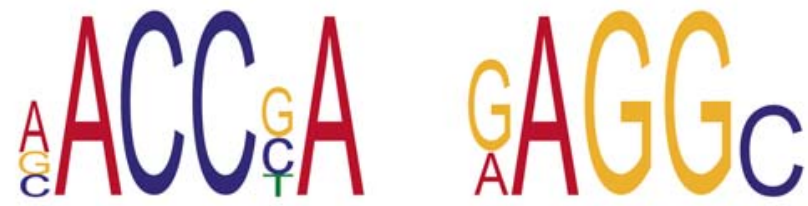

Soybean
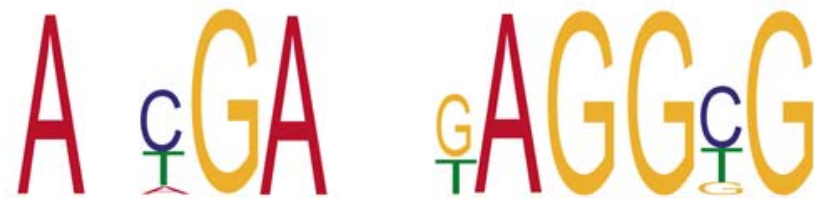

FIGURE 3 | Distribution pattern (A) and representative motifs (B) of $6 \mathrm{~mA}$ in in rice, Arabidopsis, soybean, and strawberry. Gene bodies include exons, introns, and 5 and 3' UTRs. Data were reproduced by previous studies (Liang et al., 2018b; Zhang et al., 2018; Xie et al., 2020; Yuan et al., 2020).

\section{BIOLOGICAL FUNCTION OF 6mA IN PLANTS}

$6 \mathrm{~mA}$ has been found to be positively associated with expression of key genes, which may be responsible for discrepancy in both developmental cues and stress tolerance in plants. In rice, mutations in DDM1 (Deficient in DNA Methylation 1) lead to decreased $6 \mathrm{~mA}$ levels and defects in plant growth (Zhang et al., 2018). By CRISPR/Cas9 gene editing technology, double mutant $d d m 1 a / 1 b$ show dwarf, decreased seed-setting rate and infertility phenotype compared with wild-type plants (Zhang et al., 2018). To answer the question how DDM1 regulates rice growth, this study further identified $6 \mathrm{~mA}$ modified genes that were differentially expressed in $d d m 1 a / 1 b$, such as Grain 
number, plant height, and heading date7 (GHD7), BR-Deficient Dwarf1 (BRD1), and DWARF7 (DWF7) (Zhang et al., 2018). Similarly, mutation in OSALKBH1 exhibits earlier heading date with two to threefold increases of $6 \mathrm{~mA}$ levels (Zhou et al., 2018). And also, in Arabidopsis, flowering promoters FLOWERING LOCUS T (FT) and FRUITFULL (FUL) were associated with new $6 \mathrm{~mA}$ modification, when increased their expression during floral transition (Liang et al., 2018b). Together, these examples likely imply that association between $6 \mathrm{~mA}$ modification and gene transcription may contribute to plant developmental cues.

DNA methylation control a wide range of (a)biotic stress conditions. Several studies have revealed positive correlations of DNA methylation and key genes for stress responses in plants (Popova et al., 2013; Migicovsky et al., 2014; Zheng et al., 2017). Study on $6 \mathrm{~mA}$ modification among rice cultivars from japonica and indica group revealed varied response under different environmental conditions (Zhang et al., 2018). For example, japonica cultivar (Nippobare) was found to be more tolerant to cold and salt stress while indica (93-11) more tolerant to heat stress due to difference in $6 \mathrm{~mA}$ levels. Thus, essential roles of $6 \mathrm{~mA}$ associated with long-term environmental adaptations can be exploited in further plant improvement programs.

Hence, $6 \mathrm{~mA}$ is yet an unknown DNA epigenetic modification linked to actively expressed genes in model plants Arabidopsis and rice and might play an active role in plant development and stress responses. It will be important for future studies to reveal how $6 \mathrm{~mA}$ regulates gene expression, identification and characterization of interacting proteins will help in understanding the functions and mechanisms by which $6 \mathrm{~mA}$ influences gene expression in plants, especially for nonmodel plants.

\section{6mA PREDICTORS BY MACHINE LEARNING AND DEEP LEARNING}

Although several experimental methods have been suggested for the genome-wide identification of $6 \mathrm{~mA}$ sites, these approaches are time consuming and laborious. Given that genome-wide $6 \mathrm{~mA}$ profiles have developed with single-base resolution (Liang et al., 2020), and since the distribution of the $6 \mathrm{~mA}$ sites in the genome is non-random with consensus GAGG motif resent in Arabidopsis, rice and other eukaryotic organisms that is only partially conserved (Liang et al., 2018a). A precise and reliable method for identification of $6 \mathrm{~mA}$ sites is in silico machine learning and deep learning invaluable to gain a better understanding of the regulatory mechanism of $6 \mathrm{~mA}$. Previous studies have demonstrated that deep learning is a powerful approach for sequence analysis and classification in bioinformatics (Chen et al., 2019; Lv et al., 2019; Yu and Dai, 2019). In order to identify genome wide $6 \mathrm{~mA}$ sites, several machine learning methods have recently been developed (Table 1; Feng et al., 2019; Yu and Dai, 2019; Lv et al., 2019, 2020; Pian et al., 2020; Wang et al., 2020). For example, Chen et al. recently presented a $6 \mathrm{~mA}$ benchmark dataset comprising $8806 \mathrm{~mA}$ sites and 880 non- $6 \mathrm{~mA}$ sites in the genome of rice (Chen et al., 2019), which was signified as 6mA-rice-Chen. They constructed a support vector machine (SVM) based tool called i6mA-Pred for identifying $6 \mathrm{~mA}$ sites in the rice genome by using several manually designed DNA sequence features. It was documented that on the rice genome dataset, $16 \mathrm{mAPred}$ had an accuracy of $\sim 83 \%$ (Chen et al., 2019). In another study, Pian et al. (2020) suggested a method based on the markov model (MM) for prediction of $6 \mathrm{~mA}$ sites, called MM-6mAPred. Based on the $6 \mathrm{~mA}$-rice-Chen benchmark dataset, they developed and evaluated their MM-6mAPred. MM-6mAPred has been reported to outperform i6mA-Pred in the $6 \mathrm{~mA}$ sites prediction with the sensitivity and specificity of $\sim 89$ and $\sim 90 \%$, respectively, having the overall accuracy of $\sim 90 \%$ (Pian et al., 2020). For the identification of $6 \mathrm{~mA}$ in the rice genome, Tahir et al. recommended another computational method, called iDNA6mA (Tahir et al., 2019). They also trained and evaluated their iDNA6mA on the 6mA-rice-Chen dataset, and they discovered that in prediction performance, iDNA6mA outperformed i6mAPred. A method, called SDM6A, was proposed by Basith et al. to predict $6 \mathrm{~mA}$ sites in the rice genome (Basith et al., 2019). SDM6A is an ensemble technique that uses many methods of encoding features and classifiers for machine learning. They trained and assessed their SDM6A on the basis of the $6 \mathrm{~mA}$ rice-Chen benchmark dataset, and they discovered that on the $6 \mathrm{~mA}$-rice-Chen benchmark dataset, SDM6A outperformed i6mA-Pred and iDNA6mA.

A further $6 \mathrm{~mA}$ benchmark dataset for the rice genome can be denoted as 6mA-rice- $\mathrm{Lv}$ ( $\mathrm{Lv}$ et al., 2019). It contains $154,0006 \mathrm{~mA}$ sites-contained sequences as positive samples and the same number of negative samples. They trained and analyzed iDNA6mA-rice on 6mA-rice-Lv dataset by fivefold cross-validation and found that iDNA6mA-rice had strong predictive efficiency. They also trained and tested iDNA6mArice on the $6 \mathrm{~mA}$-rice-Chen dataset for the purpose of comparison with i6mA-Pred, and found that iDNA6mArice outperformed i6mA-Pred on the 6mA-rice-Chen dataset (Lv et al., 2019). In another study a high-performance and lightweight, approach called SNNRice6mA was developed to improve the prediction accuracy of DNA $6 \mathrm{~mA}$ sites in the rice genome ( $\mathrm{Yu}$ and Dai, 2019). It is based on the architecture of convolutional neural networks. Compared to conventional machine learning approaches, it does not require a manually designed sequence feature and can learn high-level abstract features. On the $6 \mathrm{~mA}$ rice-Chen and 6mA-rice-Lv datasets, SNNRice6mA obtained an accuracy of $\sim 93$ and $92 \%$, respectively. In the prediction of DNA $6 \mathrm{~mA}$ sites in the rice genome, SNNRice6mA performed better than previous methods (Yu and Dai, 2019).

In a subsequent study a $6 \mathrm{~mA}$ predictor was trained on multi-species data (i.e., Homo sapiens (human), O. sativa (rice), Drosophila melanogaster (fruit fly), and C. elegans (worm) from a set of sequence-based features, including position-specific triple nucleotide propensity (PSTNP), physicochemical properties, and electron-ion pseudopotential interaction (EIIP). The key features were selected by performing the maximum relevance maximum distance (MRMD) analysis and the Extreme Gradient Boosting (XGBoost) algorithm was used to construct predictor. The p6mA outperformed using different datasets (Wang et al., 2020). Lv et al. (2020) in a recent study developed a computational approach for identifying $5 \mathrm{hmC}, 6 \mathrm{~mA}$, and $4 \mathrm{mC}$ sites in different species. 
TABLE 1 | Summarizing recent machine learning based $6 \mathrm{~mA}$ prediction approaches.

\begin{tabular}{|c|c|c|c|}
\hline Method name & Description & Specie & References \\
\hline i6mA-Pred & $\begin{array}{l}\text { The support vector machine approach (SVM) to identify 6mA sites in } \\
\text { rice genome with } 83 \% \text { accuracy, in which the DNA sequences are } \\
\text { effectively formulated and encoded through the use of chemical } \\
\text { property and nucleotide frequency dependent on the SVM approach }\end{array}$ & Rice genome & Chen et al., 2019 \\
\hline SNNRice6mA & $\begin{array}{l}\text { A simple and lightweight deep learning model approach for identifying } \\
6 \mathrm{~mA} \text { from rice genome, its evaluation is based on five metrics such as } \\
\text { sensitivity, accuracy, specificity, area under the curve (AUC) and } \\
\text { Matthews correlation coefficient (MCC) }\end{array}$ & Rice genome & Yu and Dai, 2019 \\
\hline i6mA-DCNP & $\begin{array}{l}\text { A high-quality computational method to identify and predict } 6 \mathrm{~mA} \text { sites } \\
\text { in the rice genome. This prediction approach is based on encoding the } \\
\text { genomic DNA samples using dinucleotides composition and the } \\
\text { optimized dinucleotide-based DNA properties }\end{array}$ & Rice genome & Kong and Zhang, 2019 \\
\hline $\begin{array}{l}\text { Sequence-based DNA } \\
\text { N6-methyladenine } \\
\text { predictor (SDM6A) }\end{array}$ & $\begin{array}{l}\text { A sequence-based two-layer method for effectively predicting novel } \\
\text { putative } 6 m A \text { sites and non- } 6 m A \text { sites in the rice genome }\end{array}$ & Rice genome & Basith et al., 2019 \\
\hline iDNA-MS & $\begin{array}{l}\text { Utilization of random forest for identifying } 6 \mathrm{~mA}, 5 \mathrm{hmC} \text {, and } 4 \mathrm{mC} \text { sites in } \\
\text { multiple species }\end{array}$ & $\begin{array}{l}\text { Multiple species, Fragaria } \\
\text { vesca, Arabidopsis thaliana, } \\
\text { Rosa chinensis, Casuarina } \\
\text { equisetifolia }\end{array}$ & Lv et al., 2020 \\
\hline Meta-i6mA & $\begin{array}{l}\text { An interspecies prediction tool to identify DNA } 6 m \text { A sites of plant } \\
\text { genome through the use of informative features in an integrative } \\
\text { machine learning framework }\end{array}$ & Rice genome & Hasan et al., 2020 \\
\hline csDMA & $\begin{array}{l}\text { A method for identifying and predicting } 6 \mathrm{~mA} \text { in various species through } \\
\text { Chou's } 5 \text {-step rule using three encoding features and different } \\
\text { algorithms to produce the feature matrix }\end{array}$ & Multiple species & Liu Z. et al., 2019 \\
\hline iDNA6mA- Rice & $\begin{array}{l}\text { The machine learning random forest algorithm to formulate the sample } \\
\text { as an input to differentiate between the methylated and non-methylated } \\
\text { sites in rice genome for evaluating } 6 \mathrm{~mA} \text { sites }\end{array}$ & Rice genome & Lv et al., 2019 \\
\hline iDNA6mA-PseKNC & $\begin{array}{l}\text { A sequence-based prediction approach that allows } 100 \% \text { accuracy } \\
\text { and } 96 \% \text { precision to identify DNA } 6 \text { mA sites without using complicated } \\
\text { mathematical formulas }\end{array}$ & Multiple species & Feng et al., 2019 \\
\hline iDNA $6 m A$ & $\begin{array}{l}\text { A deep learning method, based on the conventional neural network for } \\
\text { identifying } 6 m A \text { sites in the rice genome, which needs a single DNA } \\
\text { sequence input }\end{array}$ & Rice genome & Tahir et al., 2019 \\
\hline MM-6mA-Pred & $\begin{array}{l}\text { This tool identifies } 6 \mathrm{~mA} \text { and non-6mA sites by substantial variations in } \\
\text { transition probability among adjacent nucleotides based on Morkov's } \\
\text { model having better prediction compared to i6mA-Pred }\end{array}$ & Rice genome & Pian et al., 2020 \\
\hline DEEP6mA & $\begin{array}{l}\text { Superior performance platform to identify } 6 \mathrm{~mA} \text { sites in plants with an } \\
\text { overall prediction precision of } 94 \% \text { using a convolutional neural network } \\
\text { (CNN) to retrieve high-level sequence features and a bi-directional } \\
\text { long-term memory network (BLSTM) to acquire dependence structure } \\
\text { along the sequence }\end{array}$ & Multiple species & Li Z. et al., 2019 \\
\hline FastFeatGen & $\begin{array}{l}\text { This predictor uses a machine learning approach with motif features to } \\
\text { predict the } 6 \mathrm{~mA} \text { sites in the genome. Due to the multi-threading and } \\
\text { shared memory mechanism, speed is the advantage of this tool }\end{array}$ & Multiple species & Khaledur Rahman, 2019 \\
\hline eRice & $\begin{array}{l}\text { This prediction tool uses a machine learning approach to predict the } \\
6 \mathrm{~mA} \text { sites in the rice genome }\end{array}$ & Rice genome & Zhang et al., 2020 \\
\hline
\end{tabular}

Initially, in order to formulate samples, they used the K-tuple nucleotide portion, mono-nucleotide binary encoding scheme, and nucleotide chemical property and nucleotide frequency. Random forest was subsequently used to recognize $5 \mathrm{hmC}$, $6 \mathrm{~mA}$, and $4 \mathrm{mC}$ sites. Cross-validated results revealed that in defining the sites of three modification, the proposed approach could generate an outstanding generalization ability. Finally, a predictor called iDNA-MS was developed based on the proposed techniques (Lv et al., 2020). Zhang et al. developed AI based $6 \mathrm{~mA}$ prediction approach named the eRice database dedicated for providing reliable and efficient genomic and epigenomic resources for both japonica and indica rice (Zhang et al., 2020). Very recently Hasan et al. developed a machine learning method for predicting $6 \mathrm{~mA}$ sites in different plant genomes called Meta-i6mA. In an extensive independent test Metai6mA outperformed and revealed high Matthews correlation coefficient values of $0.918,0.827$, and 0.635 for Rosaceae, rice and Arabidopsis thaliana, respectively (Hasan et al., 2020). Currently, several machine learning approaches have been developed for identifying genome wide $6 \mathrm{~mA}$ sites, each one has their own limitations. However, there is room for their efficiency to be enhanced to predict $6 \mathrm{~mA}$ sites in various species. 


\section{CONCLUDING REMARKS AND FUTURE PERSPECTIVE}

Now, we are known that $6 \mathrm{~mA}$ has been identified as an important epigenetic mark in both mammals and model plants as the detection approaches have begun to become increasingly sensitive (Liang et al., 2020). However, it will be of importance to study the presence of $6 \mathrm{~mA}$ across the life cycle from model plants to non-model crops. As horticultural plants often equip with differentiation of cell types or organs and exhibit annual or perennial growth behaviors, using combination of rapidly growing detection approaches, it will be important to identify the biological functions of $6 \mathrm{~mA}$ and its genome localization patterns in different crops. The outstanding question is whether the biological functions of $6 \mathrm{~mA}$ in model plants are conserved in plants or it has evolved new biological functions in these organisms.

As $6 \mathrm{~mA}$ occurs less frequently in more recently evolved mammals and plants, this might reflect a more specialized functional role in crops. Moreover, in model plants, we have known $6 \mathrm{~mA}$ functions as a complimentary epigenetic mark to $5 \mathrm{mC}$ that is in contrast with fungi where the strong negative relation is observed between $6 \mathrm{~mA}$ and $5 \mathrm{mC}$ (Zhang et al., 2018; Liang et al., 2020). In addition, it has been revealed that $6 \mathrm{~mA}$ is associated with histone modifications in mammals (Greer et al., 2015; Ma et al., 2019). So, it will be of interest to further investigate their interactions and cross-talk signals between $6 \mathrm{~mA}$ and other types of epigenetic marks in plants and identify weather they combinedly regulate gene activity and function (Figure 1C). With the advancement in detection approaches of

\section{REFERENCES}

Allis, C. D., and Jenuwein, T. (2016). The molecular hallmarks of epigenetic control. Nat. Rev. Genet. 17, 487-500. doi: 10.1038/nrg.2016.59

Basith, S., Manavalan, B., Shin, T. H., and Lee, G. (2019). SDM6A: a web-based integrative machine-learning framework for predicting $6 \mathrm{~mA}$ sites in the rice genome. Mol. Ther. Nucleic Acids 18, 131-141. doi: 10.1016/j.omtn.2019.08.011

Chen, K., Lu, Z., Wang, X., Fu, Y., Luo, G. Z., Liu, N., et al. (2015). Highresolution N6-methyladenosine $\left(\mathrm{m}^{6} \mathrm{~A}\right)$ map using photo-crosslinking-assisted m6A sequencing. Angew. Chemie Int. Ed. 54, 1587-1590. doi: 10.1002/anie. 201410647

Chen, W., Lv, H., Nie, F., Lin, H., and Hancock, J. (2019). I6mA-Pred: identifying DNA N6-methyladenine sites in the rice genome. Bioinformatics 35, 2796-2800. doi: 10.1093/bioinformatics/btz015

Cheng, J., Niu, Q., Zhang, B., Chen, K., Yang, R., Zhu, J. K., et al. (2018). Downregulation of RdDM during strawberry fruit ripening. Genome Biol. 19:212. doi: 10.1186/s13059-018-1587-x

Duan, C. G., Zhu, J. K., and Cao, X. (2018). Retrospective and perspective of plant epigenetics in China. J. Genet. Genomics 45, 621-638. doi: 10.1016/j.jgg.2018. 09.004

Fang, G., Munera, D., Friedman, D. I., Mandlik, A., Chao, M. C., Banerjee, O., et al. (2012). Genome-wide mapping of methylated adenine residues in pathogenic Escherichia coli using single-molecule real-time sequencing. Nat. Biotechnol. 30, 1232-1239. doi: 10.1038/nbt.2432

Feng, P., Yang, H., Ding, H., Lin, H., Chen, W., and Chou, K. C. (2019). iDNA6mAPseKNC: identifying DNA N 6 -methyladenosine sites by incorporating nucleotide physicochemical properties into PseKNC. Genomics 111, 96-102. doi: 10.1016/j.ygeno.2018.01.005

Feng, S., Cokus, S. J., Zhang, X., Chen, P.-Y. P.-Y., Bostick, M., Goll, M. G., et al. (2010). Conservation and divergence of methylation patterning in plants
$6 \mathrm{~mA}$, future studies should focus on the environmental factors or combine with specific developmental cues regulated by $6 \mathrm{~mA}$ in horticultural plants and its conserved/divergent enzymes at different species level, which will offer clues for its biological significance and evolutionary conservation.

In addition, as deep learning approaches have become a powerful strategy for modeling and prediction "Big Data" in genomics and epigenomics (including $6 \mathrm{~mA}$ ), from our perspective, it will lead to detailed and comprehensive detection of $6 \mathrm{~mA}$, its potential functions and localization in diverse plant species, especially for horticultural crops. Thus, detection, identification, and prediction of $6 \mathrm{~mA}$ in non-model crops using latest sensitive detection methods that developed in model plants will open up a new and exciting chapter of discovery in the field of DNA methylation in plants.

\section{AUTHOR CONTRIBUTIONS}

SL and CG conceived and designed this review. SC and JL drafted the manuscript and created the figures. PZ and AR helped to reviewing and editing the manuscript. All authors have read and agreed to the published version of the manuscript.

\section{FUNDING}

This research was supported by the Special Project of Science and Technology Innovation Plan of Shaanxi Academy of Forestry (SXLK2020-0212) and National Key Research and Development Program of China (2019YFD1000600).

and animals. Proc. Natl. Acad. Sci. U.S.A. 107, 8689-8694. doi: 10.1073/pnas. 1002720107

Flusberg, B. A., Webster, D. R., Lee, J. H., Travers, K. J., Olivares, E. C., Clark, T. A., et al. (2010). Direct detection of DNA methylation during single-molecule, real-time sequencing. Nat. Methods 7, 461-465. doi: 10.1038/nmeth.1459

Fu, Y., Luo, G.-Z. Z., Chen, K., Deng, X., Yu, M., Han, D., et al. (2015). N6-methyldeoxyadenosine marks active transcription start sites in Chlamydomonas. Cell 161, 879-892. doi: 10.1016/j.cell.2015.04.010

Gallego-Bartolomé, J., Gardiner, J., Liu, W., Papikian, A., Ghoshal, B., Kuo, H. Y. H. Y., et al. (2018). Targeted DNA demethylation of the Arabidopsis genome using the human TET1 catalytic domain. Proc. Natl. Acad. Sci. U.S.A. 115:201716945. doi: 10.1073/pnas.1716945115

Gallusci, P., Dai, Z., Génard, M., Gauffretau, A., Leblanc-Fournier, N., RichardMolard, C., et al. (2017). Epigenetics for plant improvement: current knowledge and modeling avenues. Trends Plant Sci. 22, 610-623. doi: 10.1016/j.tplants. 2017.04.009

Greer, E. L., Blanco, M. A., Gu, L., Sendinc, E., Liu, J., Aristizábal-Corrales, D., et al. (2015). DNA methylation on N6-adenine in C. elegans. Cell 161, 868-878. doi: 10.1016/j.cell.2015.04.005

Hasan, M. M., Basith, S., Khatun, M. S., Lee, G., Manavalan, B., and Kurata, H. (2020). Meta-i6mA: an interspecies predictor for identifying DNA N6methyladenine sites of plant genomes by exploiting informative features in an integrative machine-learning framework. Brief. Bioinform. bbaa202. doi: 10.1093/bib/bbaa202

Heard, E., and Martienssen, R. A. (2014). Transgenerational epigenetic inheritance: myths and mechanisms. Cell 157, 95-109. doi: 10.1016/j.cell.2014.02.045

Huang, H., Liu, R., Niu, Q., Tang, K., Zhang, B., Zhang, H., et al. (2019). Global increase in DNA methylation during orange fruit development and ripening. Proc. Natl. Acad. Sci. U.S.A. 116, 1430-1436. doi: 10.1073/pnas.181544 1116 
Huang, W., Xiong, J., Yang, Y., Liu, S.-M., Yuan, B.-F., and Feng, Y.-Q. (2015). Determination of DNA adenine methylation in genomes of mammals and plants by liquid chromatography/mass spectrometry. RSC Adv. 5, 64046-64054. doi: 10.1039/C5RA05307B

Khaledur Rahman, M. (2019). FastFeatGen: faster parallel feature extraction from genome sequences and efficient prediction of DNA N6-methyladenine sites. bioRxiv [preprint] bioRxiv: 846311. doi: 10.1101/846311.

Kong, L., and Zhang, L. (2019). I6mA-DNCP: computational identification of DNA N6-methyladenine sites in the rice genome using optimized dinucleotide-based features. Genes (Basel) 10:828. doi: 10.3390/genes10100828

Lang, Z., Wang, Y., Tang, K., Tang, D., Datsenka, T., Cheng, J., et al. (2017). Critical roles of DNA demethylation in the activation of ripening-induced genes and inhibition of ripening-repressed genes in tomato fruit. Proc. Natl. Acad. Sci. U.S.A. 114, E4511-E4519. doi: 10.1073/pnas.1705233114

Li, Z., Jiang, H., Kong, L., Chen, Y., Zhang, L., and Pian, C. (2019). Deep6mA: a deep learning framework for exploring similar patterns in DNA N6methyladenine sites across different species. bioRxiv 17:889824. doi: 10.1101/ 2019.12.28.889824

Liang, Z., Geng, Y., and Gu, X. (2018a). Adenine methylation: new epigenetic marker of DNA and mRNA. Mol. Plant 11, 1219-1221. doi: 10.1016/j.molp. 2018.08.001

Liang, Z., Riaz, A., Chachar, S., Ding, Y., Du, H., and Gu, X. (2020). Epigenetic modifications of mRNA and DNA in plants. Mol. Plant 13, 14-30. doi: 10.1016/ j.molp.2019.12.007

Liang, Z., Shen, L., Cui, X., Bao, S., Geng, Y., Yu, G., et al. (2018b). DNA N6Adenine Methylation in Arabidopsis thaliana. Dev. Cell 45, 406-416. doi: 10. 1016/j.devcel.2018.03.012

Liang, Z., Zhang, Q., Ji, C., Hu, G., Zhang, P., Wang, Y., et al. (2021). Reorganization of the $3 \mathrm{D}$ chromatin architecture of rice genomes during heat stress. BMC Biol. 19:53. doi: 10.1186/s12915-021-00996-4

Liu, Z., Dong, W., Jiang, W., and He, Z. (2019). csDMA: an improved bioinformatics tool for identifying DNA $6 \mathrm{~mA}$ modifications via Chou's 5-step rule. Sci. Rep. 9:13109. doi: 10.1038/s41598-019-49430-4

Liu, Z. Y., Xing, J. F., Chen, W., Luan, M. W., Xie, R., Huang, J., et al. (2019). MDR: an integrative DNA N6-methyladenine and N4-methylcytosine modification database for Rosaceae. Hortic. Res. 6:78. doi: 10.1038/s41438-019-0160-4

Luo, G. Z., Macqueen, A., Zheng, G., Duan, H., Dore, L. C., Lu, Z., et al. (2014). Unique features of the m6A methylome in Arabidopsis thaliana. Nat. Commun. 5:5630. doi: $10.1038 /$ ncomms6630

Lv, H., Dao, F. Y., Guan, Z. X., Zhang, D., Tan, J. X., Zhang, Y., et al. (2019). iDNA6mA-rice: a computational tool for detecting N6-Methyladenine sites in rice. Front. Genet. 10:793. doi: 10.3389/fgene.2019.00793

Lv, H., Dao, F. Y., Zhang, D., Guan, Z. X., Yang, H., Su, W., et al. (2020). iDNA-MS: an integrated computational tool for detecting DNA modification sites in multiple genomes. iScience 23:100991. doi: 10.1016/j.isci.2020.10 0991

Ma, C., Niu, R., Huang, T., Shao, L., Peng, Y., Ding, W., et al. (2019). N6methyldeoxyadenine is a transgenerational epigenetic signal for mitochondrial stress adaptation. Nat. Cell Biol. 21, 319-327. doi: 10.1038/s41556-018-0238-5

Migicovsky, Z., Yao, Y., and Kovalchuk, I. (2014). Transgenerational phenotypic and epigenetic changes in response to heat stress in Arabidopsis thaliana. Plant Signal. Behav. 9:e27971. doi: 10.4161/psb.27971

Mozgova, I., and Hennig, L. (2015). The polycomb group protein regulatory network. Annu. Rev. Plant Biol. 66, 269-296. doi: 10.1146/annurev-arplant043014-115627

Niederhuth, C. E., and Schmitz, R. J. (2017). Putting DNA methylation in context: from genomes to gene expression in plants. Biochim. Biophys. Acta Gene Regul. Mech. 1860, 149-156. doi: 10.1016/j.bbagrm.2016.08.009

O'Brown, Z. K., and Greer, E. L. (2016). N6-methyladenine: a conserved and dynamic DNA mark. Adv. Exp. Med. Biol. 945, 213-246. doi: 10.1007/978-3319-43624-1_10

Pian, C., Zhang, G., Li, F., Fan, X., and Hancock, J. (2020). MM-6mAPred: identifying DNA N6-methyladenine sites based on Markov model. Bioinformatics 36, 388-392. doi: 10.1093/bioinformatics/btz556

Popova, O. V., Dinh, H. Q., Aufsatz, W., and Jonak, C. (2013). The RdDM pathway is required for basal heat tolerance in Arabidopsis. Mol. Plant 6, 396-410. doi: $10.1093 / \mathrm{mp} / \mathrm{sst} 023$

Sabbione, A., Daurelio, L., Vegetti, A., Talón, M., Tadeo, F., and Dotto, M. (2019). Genome-wide analysis of $A G O, D C L$ and $R D R$ gene families reveals
RNA-directed DNA methylation is involved in fruit abscission in Citrus sinensis. BMC Plant Biol. 19:401. doi: 10.1186/s12870-019-1998-1

Smith, Z. D., and Meissner, A. (2013). DNA methylation: roles in mammalian development. Nat. Rev. Genet. 14, 204-220. doi: 10.1038/nrg3354

Springer, N. M., and Schmitz, R. J. (2017). Exploiting induced and natural epigenetic variation for crop improvement. Nat. Rev. Genet. 18, 563-575. doi: 10.1038/nrg.2017.45

Tahir, M., Tayara, H., and Chong, K. T. (2019). iDNA6mA (5-step rule): identification of DNA N6-methyladenine sites in the rice genome by intelligent computational model via Chou's 5-step rule. Chemom. Intell. Lab. Syst. 189, 96-101. doi: 10.1016/j.chemolab.2019.04.007

Tang, D., Gallusci, P., and Lang, Z. (2020). Fruit development and epigenetic modifications. New Phytol. 228, 839-844. doi: 10.1111/nph.16724

Usai, G., Vangelisti, A., Simoni, S., Giordani, T., Natali, L., and Cavallini, A. (2021). DNA modification patterns within the transposable elements of the fig (Ficus carica L .) genome. Plants 10:451. doi: 10.3390/plants10030451

Wang, H. T., Xiao, F. H., Li, G. H., and Kong, Q. P. (2020). Identification of DNA N6-methyladenine sites by integration of sequence features. Epigenetics Chromatin. 13:8. doi: 10.1186/s13072-020-00330-2

Xiao, C., Yan, G.-R., Xie, S.-Q., Gu, X.-F., Chen, Y., Bo, X.-C., et al. (2018). N6-methyladenine DNA modification in the human genome. Mol. Cell 71, 306-318.e7. doi: 10.1016/j.molcel.2018.06.015

Xie, S. Q., Xing, J. F., Zhang, X. M., Liu, Z. Y., Luan, M. W., Zhu, J., et al. (2020). N6Methyladenine DNA modification in the woodland strawberry (Fragaria vesca) genome reveals a positive relationship with gene transcription. Front. Genet. 10:1288. doi: 10.3389/fgene.2019.01288

Ye, G., Zhang, H., Chen, B., Nie, S., Liu, H., Gao, W., et al. (2019). De novo genome assembly of the stress tolerant forest species Casuarina equisetifolia provides insight into secondary growth. Plant J. 97, 779-794. doi: 10.1111/tpj.14159

Yu, H., and Dai, Z. (2019). SNNRice6mA: a deep learning method for predicting dna n6-methyladenine sites in rice genome. Front. Genet. 10:1071. doi: 10.3389/ fgene.2019.01071

Yuan, D. H., Xing, J. F., Luan, M. W., Ji, K. K., Guo, J., Xie, S. Q., et al. (2020). DNA N6-methyladenine modification in wild and cultivated soybeans reveals different patterns in nucleus and cytoplasm. Front. Genet. 11:736. doi: 10.3389/ fgene.2020.00736

Zhang, P., Wang, Y., Chachar, S., Tian, J., and Gu, X. (2020). eRice: a refined epigenomic platform for japonica and indica rice. Plant Biotechnol. J. 18, 1642-1644. doi: 10.1111/pbi.13329

Zhang, Q., Liang, Z., Cui, X., Ji, C., Li, Y., Zhang, P., et al. (2018). N6-methyladenine DNA methylation in japonica and indica rice genomes and its association with gene expression, plant development, and stress responses. Mol. Plant 11, 1492-1508. doi: 10.1016/j.molp.2018.11.005

Zheng, X., Chen, L., Xia, H., Wei, H., Lou, Q., Li, M., et al. (2017). Transgenerational epimutations induced by multi-generation drought imposition mediate rice plant's adaptation to drought condition. Sci. Rep. 7, 39843. doi: 10.1038/srep39843

Zhou, C., Wang, C., Liu, H., Zhou, Q., Liu, Q., Guo, Y., et al. (2018). Identification and analysis of adenine N6-methylation sites in the rice genome. Nat. Plants 4, 554-563. doi: 10.1038/s41477-018-0214-X

Zhou, C., Yuan, Z., Ma, X., Yang, H., Wang, P., Zheng, L., et al. (2021). Accessible chromatin regions and their functional interrelations with gene transcription and epigenetic modifications in sorghum genome. Plant Commun. 2:100140. doi: 10.1016/j.xplc.2020.100140

Zhu, S., Beaulaurier, J., Deikus, G., Wu, T. P., Strahl, M., Hao, Z., et al. (2018). Mapping and characterizing N6-methyladenine in eukaryotic genomes using single-molecule real-time sequencing. Genome Res. 28, 1067-1078. doi: 10. $1101 /$ gr.231068.117

Conflict of Interest: The authors declare that the research was conducted in the absence of any commercial or financial relationships that could be construed as a potential conflict of interest.

Copyright (C) 2021 Chachar, Liu, Zhang, Riaz, Guan and Liu. This is an open-access article distributed under the terms of the Creative Commons Attribution License (CC BY). The use, distribution or reproduction in other forums is permitted, provided the original author(s) and the copyright owner(s) are credited and that the original publication in this journal is cited, in accordance with accepted academic practice. No use, distribution or reproduction is permitted which does not comply with these terms. 